\title{
Nuevo modelo de clasificación de revistas científicas colombianas -PUBLINDEX- Panorama de la publicación científica en Colombia
}

\author{
The new classification model of Colombian scientific journals \\ -PUBLINDEX-Scientific publication outlook in Colombia
}

\section{Novo modelo de classificação das revistas científicas da Colômbia -PUBLINDEX-Panorama da publicação científica na Colômbia}

El nuevo modelo de clasificación de revistas de PUBLINDEX y su política fue socializado durante el mes de junio de 2016 en distintas ciudades de Colombia. En esta socialización se presentó el índice $\mathrm{H} 5$ como el nuevo método de medición del impacto de las revistas que sería utilizado por PUBLINDEX. Además, se presentaron proyecciones para definir los posibles escenarios que permitieran determinar los puntos de corte para cada una de las categorías de la clasificación de las revistas colombianas.
Estas proyecciones fueron calculadas para una ventana de 6 años de observación (2009-2014 - Tabla 1) y no para 5 años de observación, el cual, por ser un período más amplio aumenta el valor del índice $\mathrm{H}$ " 5 "; además, existe otro factor que influye en que el cálculo del índice $\mathrm{H}$ sea mayor y es la antigüedad del período evaluado que parte del año 2009, por lo que las proyecciones realizadas en ese período específico serán mayores a las realizadas en un período de 5 años más reciente como 2011-2015 o 2012-2016(1).

Tabla 1. Proyecciones PUBLINDEX 2009-2014, presentado en la socialización del nuevo modelo de clasificación en junio de 2016.

\begin{tabular}{|c|c|c|c|c|c|c|c|c|c|c|c|}
\hline & \multirow{2}{*}{\multicolumn{2}{|c|}{$\begin{array}{c}\text { CIENCIAS } \\
\text { DE LA VIDA }\end{array}$}} & \multirow{2}{*}{\multicolumn{2}{|c|}{ HUMANIDADES }} & \multirow{2}{*}{\multicolumn{2}{|c|}{ INGENIERIAA }} & \multirow{2}{*}{\multicolumn{2}{|c|}{$\begin{array}{c}\text { PURAS Y } \\
\text { APLICADAS }\end{array}$}} & \multirow{2}{*}{\multicolumn{2}{|c|}{$\begin{array}{l}\text { CIENCIAS } \\
\text { SOCIALES }\end{array}$}} & \multirow{5}{*}{$\begin{array}{l}\text { Total de } \\
\text { revistas }\end{array}$} \\
\hline & & & & & & & & & & & \\
\hline & Rang & Número & Rang & Número & Rang & Número & Rang & Número & Rang & Número & \\
\hline & 0 & de & 0 & de & 0 & de & 0 & de & 0 & de & \\
\hline & I H5 & Revistas & I H5 & Revistas & I H5 & Revistas & I H5 & Revistas & I H5 & Revistas & \\
\hline Q1 & $9-18$ & 14 & $8-10$ & 10 & $7-12$ & 7 & $9-22$ & 6 & $9-21$ & 33 & $\underline{70}$ \\
\hline Q2 & $6-8$ & 19 & $5-7$ & 11 & $6-6$ & 3 & $7-8$ & 12 & $7-8$ & 21 & $\underline{66}$ \\
\hline Q3 & $5-5$ & 12 & $4-4$ & 6 & $5-5$ & 6 & $5-6$ & 8 & $5-6$ & 45 & $\underline{77}$ \\
\hline Q4 & $3-4$ & 23 & $3-3$ & 15 & $3-4$ & 11 & $3-4$ & 14 & $3-4$ & 53 & $\underline{116}$ \\
\hline & & $\underline{68}$ & & 42 & & $\underline{27}$ & & $\underline{40}$ & & 152 & $\underline{329}$ \\
\hline
\end{tabular}


En agosto de 2016 se publicó el modelo definitivo de clasificación de las revistas, en el que se definió como requisito para que una revista obtenga la categoría A1 y A2, el estar ubicada en el intercuartil Q1 y Q2 del Journal Citation Report (JCR) o del SCImago Journal \& Country Rank (SJR) respectivamente; para estar en la categoría B, el criterio estar ubicada en el intercuartil Q3 de JCR-SJR o estar ubicada en el intercuartil Q1 del índice H5; y para la categoría C, el criterio es estar ubicada en el intercuartil Q4 de JCR-SJR o estar ubicada en el intercuartil Q2 del índice $\mathrm{H} 5$. El criterio de ingreso para ser clasificado en el índice H5 es el de un punto de corte mínimo de 2 para el período 20122016(2).

De acuerdo con las proyecciones realizadas en las charlas de socialización, y aplicando el modelo definitivo publicado en agosto de 2016, podrían entrar a ser parte de los intercuartiles del Îndice H5 un total de 329 revistas. Entre ellas, 193 revistas no cumplirían el requisito mínimo para llegar a ser indexadas en al menos la categoría $\mathrm{C}$, quedando entonces indexadas solo 136 revistas, que estarían ubicadas entre los intercuartiles Q1 y Q2 del índice H5 (Tabla 1). Es necesario tener en cuenta que el índice $\mathrm{H}$ de estas revistas se basa en el cálculo del período 2009-2014 (6 años) y no del modelo final que será del período 2012-2016 (5 años). Este último modelo se caracteriza por tener un año menos de observación y ser un período más reciente, lo cual hace que el índice $\mathrm{H}$ calculado sea menor, provocando probablemente que el número final de revistas indexadas sea incluso menor al de las proyecciones iniciales de Publindex (1-2).

El panorama que muestra PUBLINDEX para las revistas colombianas y en especial a las revistas de libre acceso que cuentan con menos recursos que las revistas pagas es caótico, puesto que significa la pérdida de clasificación de aproximadamente el $75 \%$ de las revistas del país que para el año 2014 se encontraban indexadas 542 (2).

Esto puede ocasionar que todos los futuros manuscritos sean sometidos en las revistas que hayan quedado clasificadas, provocando la saturación del proceso editorial de dichas revistas; esta situación favorecerá a aquellas revistas pagas las cuales por este aumento de volumen podrán continuar con sus procesos editoriales aumentando su personal. Por el contrario, las revistas de libre acceso cuyo funcionamiento se basa en el trabajo ad honorem de estudiantes e investigadores, muy probablemente no podrán contar con una fuente de financiación extra, lo que las colocaría en clara desventaja (3).

Por otro lado, el aumento del volumen de manuscritos sometidos a las pocas revistas indexadas de libre acceso ocasionaría que el proceso editorial sufra atrasos, y disminuiría la rapidez de la difusión científica de los investigadores que decidan publicar en estas revistas. En estas condiciones, no sería descabellada la perspectiva de que desaparezcan en Colombia las revistas de libre acceso, y la única opción que tendrían los investigadores sería la de buscar publicar en revistas extranjeras de libre acceso de países desarrollados que cuentan con el personal y los fondos suficientes para la labor editorial(3).

Esta situación también puede llevar al peor escenario posible: en donde para poder mantener este índice $\mathrm{H}$ elevado se incurra en realizar citaciones de "cortesía" entre los contactos de las revistas. Este posible escenario sería el peor para el desarrollo de la ciencia de nuestro país, pero sería un recurso obligado por la necesidad del $75 \%$ de revistas que quedarían sin ser clasificadas por Colciencias para intentar sobrevivir al nuevo modelo de clasificación. Si este escenario llegara a ocurrir, la autonomía editorial se perdería en gran medida y además se podría poner en tela de juicio la calidad de las publicaciones nacionales.

Julián Camilo Cala Duran, MD. Investigador Grupo de Investigaciones Clínicas UNAB, Grupo de Investigación CARING UNAB Correo electrónico: jcala2@unab.edu.co

\section{Referencias}

1. Socialización y Discusión de la política, Bucaramanga, junio 10 de 2016.

2. PUBLINDEX-COLCIENCIAS. Política Nacional para Mejorar el Impacto de las Publicaciones Científicas Nacionales, Documento N. ${ }^{\circ} 1601$. Dirección de fomento a la Investigación. Agosto de 2016.

3. Ríos-González CM, Carvajal-Tapia AE. El rol de las revistas científicas estudiantiles en la difusión de conocimientos en pregrado. MedUNAB 2016; 19 (1): 78. 